\title{
A Fully Optical Ring Network-on-Chip with Static and Dynamic Wavelength Allocation
}

\author{
Ahmadou Dit Adi CISSE ${ }^{\dagger \mathrm{a})}$, Nonmember, Michihiro KOIBUCHI ${ }^{\dagger \dagger \mathrm{b})}$, Masato YOSHIMI ${ }^{\dagger c)}$, Hidetsugu IRIE $^{\dagger \mathrm{d})}$, \\ and Tsutomu YOSHINAGA ${ }^{\dagger \mathrm{e})}$, Members
}

\begin{abstract}
SUMMARY Silicon photonics Network-on-Chips (NoCs) have emerged as an attractive solution to alleviate the high power consumption of traditional electronic interconnects. In this paper, we propose a fully optical ring NoC that combines static and dynamic wavelength allocation communication mechanisms. A different wavelength-channel is statically allocated to each destination node for light weight communication. Contention of simultaneous communication requests from multiple source nodes to the destination is solved by a token based arbitration for the particular wavelength-channel. For heavy load communication, a multiwavelength-channel is available by requesting it in execution time from source node to a special node that manages dynamic allocation of the shared multiwavelength-channel among all nodes. We combine these static and dynamic communication mechanisms in a same network that introduces selection techniques based on message size and congestion information. Using a photonic NoC simulator based on Phoenixsim, we evaluate our architecture under uniform random, neighbor, and hotspot traffic patterns. Simulation results show that our proposed fully optical ring NoC presents a good performance by utilizing adequate static and dynamic channels based on the selection techniques. We also show that our architecture can reduce by more than half, the energy consumption necessary for arbitration compared to hybrid photonic ring and mesh NoCs. A comparison with several previous works in term of architecture hardware cost shows that our architecture can be an attractive cost-performance efficient interconnection infrastructure for future SoCs and CMPs.

key words: Network-on-Chip, high-bandwidth and low power network, optical interconnect, wavelength allocation
\end{abstract}

\section{Introduction}

As the number of cores on a chip increases, many-core and system-on-chips (SoCs) interconnections will require high performance and low power consumption. Traditional electronic network-on-chip (NoC) faces several problems, such as limited bandwidth, crosstalk, impedance mismatch, and huge power dissipation. Photonic communication technology offers an opportunity to reduce the interconnection power consumption while meeting future chip multiprocessors (CMPs) performance requirements. It has attracted attention with recent advances on development of required

\footnotetext{
Manuscript received January 11, 2013.

Manuscript revised May 1, 2013.

${ }^{\dagger}$ The authors are with The University of Electro-Communications, Chofu-shi, 182-8585 Japan.

${ }^{\dagger \dagger}$ The author is with National Institute of Informatics, Tokyo, 101-8430 Japan.

a)E-mail: ahmadou@comp.is.uec.ac.jp

b)E-mail: koibuchi@nii.ac.jp

c) E-mail: yoshimi@is.uec.ac.jp

d)E-mail: irie@is.uec.ac.jp

e)E-mail: yosinaga @is.uec.ac.jp

DOI: $10.1587 /$ transinf.E96.D.2545
}

silicon photonics devices. CMOS-compatible micro-ring resonators (MRs [1], [2]), photonic detectors [3], and silicon waveguides [4], [5] are key devices, which are able to integrate photonic network at the chip level. Several researches that combine photonic and electronic interconnects (hybrid NoCs [6]-[11]) or use pure optical interconnects (fully optical NoCs [12]-[15]) showed that silicon photonics could be a promising solution for future NoCs. In this work we propose a new photonic NoC that addresses the following issues:

- Hybrid NoCs suffer from high power consumption of either electronic path setup networks [6], [7], [11] or local communication using electronic interconnects [8][10].

- Previously proposed photonic NoCs in one hand use only low bandwidth static wavelength allocation [9], [12], [14], [15], with short or without arbitration overhead. On the other hand, photonic NoCs with only high bandwidth dynamic wavelength allocation suffer of higher arbitration overhead [10], [13].

Our architecture has the advantage of being a fully optical, low power NoC, that can employ static and dynamic wavelength allocation techniques in the same network. It consists of optical switches connected using three waveguides in a multi-ring topology. These rings of waveguides are used for static and dynamic wavelength allocation communications, and arbitration respectively.

For the first waveguide, used for static wavelength allocation communication, a single wavelength-channel is allocated to each network node. Contention of simultaneous communication requests from multiple source nodes to the destination is solved by a token based arbitration for the particular wavelength-channel.

In the second waveguide that uses dynamic wavelength allocation communication; multiple wavelengths are allocated to a single source-destination pair by a manager node. It provides high-bandwidth communication by making good use of wavelength-division multiplexing (WDM). The manager node is a particular node which provides arbitration capabilities. It receives requests from source nodes, and arbitrates the allocation of the dynamic communication waveguide's wavelengths. While static wavelength allocation has short arbitration overhead since an independent wavelengthchannel is assigned to each destination node, it provides lower data transfer bandwidth compared to the dynamic 
wavelength allocation. However, the dynamic wavelength allocation mechanism needs higher overhead to establish communication.

The third waveguide is used for arbitration. It contains the tokens that represent the right to modulate data on the statically allocated wavelength-channels of static communication waveguide. Tokens are passed around the nodes to offer global arbitration mechanism.

Our architecture takes advantage of both wavelength allocation mechanisms by selecting the adequate one depending on communication message sizes (normal selection) or waveguides utilization (smart selection).

In summary, our paper has proposed the fully optical ring $\mathrm{NoC}$ which has the following advantages:

- It allows considerable reduction of the network energy consumption when compared to conventional hybrid NoCs.

- The proposal combines static and dynamic wavelength allocation mechanisms. It takes advantage of both allocation mechanisms using communication selection techniques based on message size (normal) or congestion information (smart).

We evaluate our proposed architecture using a photonic NoC simulator. Simulation results show that our architecture achieves reasonable performance under uniform random, hotspot and neighbor traffic patterns with saving energy of arbitration.

The rest of the paper is organized as follows. Section 2 briefly presents some related works. Section 3 introduces our proposed fully photonic NoC architecture. Section 4 discusses some implementation issues. Section 5 evaluates our proposal under various probabilistic traffic patterns, in terms of latency, and bandwidth. We also show analysis of energy consumption for required arbitration schemes and a hardware cost comparison of several photonic NoCs. Finally, Sect. 6 concludes and presents the future works.

\section{Related Works}

Several NoC architectures that use photonic interconnects have been proposed. The proposed architectures can be classified into two groups: hybrid [7], [9]-[11] and fully optical NoCs [13], [15].

Shacham et al. proposed a hybrid NoC that combines a broadband photonic circuit-switched network with an electronic overlay packet-switched control network [7]. The architecture uses 2- $D$ planar topologies (mesh, torus). While a direct topology is easy to scale for electronic NoCs, hybrid structures suffer from several limitations: i) huge area cost (electronic network overlaid by a similar topology photonic one); ii) hop-by-hop electronic path setup latency quickly grows with node count increases; iii) $5 \times 5$ optical switches, which are components to build the optical data transfer layer, cause several waveguide crossings and power losses.

Pan et al. proposed FIREFLY in [10], a clustered architecture with a dragonfly network topology in which nodes in the same cluster are connected by a conventional electronic interconnects, while nodes from different clusters are connected by an optical crossbar. A reservation-assisted singlewrite-multi-read resolves contention for using the optical channels. Although firefly provides high bandwidth and low power consumption photonic communication for inter clusters, it still uses electronic communication for nodes within the clusters.

PROPEL is a 64 core NoC that strikes a balance between electronic and photonic interconnects [9]. Nanophotonic interconnects are used for long distance interrouter communications, while electronic switching and flow control are used for nodes within the same tile. In addition of using different topology from FIREFLY, PROPEL statically allocates optical channels for long interrouter communications.

We proposed OREX, a hybrid NoC consisting of an optical ring and an electronic central router [11]. It uses a ring topology more adapted for optical interconnects (elimination of waveguide crossing). The setup network uses a central electronic crossbar to shorten path setup latency. OREX takes advantages of both electrical and optical technologies to offer high bandwidth with acceptable power consumption cost. However, as all hybrid architectures, the electronic interconnects still increases power consumption, although the size of required electronic control message is small. Unlike hybrid architectures, our new proposal has the advantage to be fully optical with intrinsic low power consumption.

Ventrease et al. also proposed a fully optical $\mathrm{NoC}$ called CORONA [13]. It comprises of 256 general purpose cores, organized in 64 four-core clusters. A fully optical crossbar is used to connect the clusters. Arbitration for accessing the resources can cause long data transfer delay.

Koohi et al. proposed an all-optical wavelength routed architecture [15]. Using passive routing of optical data streams based on their wavelength, the architecture eliminates the need for optical resource reservation. Unfortunately, bandwidth performance is limited due to the allocation of wavelengths to specific source-destination pairs. Our proposal in this paper offers a dual allocation mechanism; that is static wavelength allocation with low arbitration delay but low bandwidth, and a dynamic one with higher arbitration delay at the cost of extra arbitration overhead. The architecture leverages the strength of both allocation mechanisms using a selection based on message size (normal) and congestion (smart).

\section{Proposed NoC Architecture.}

\subsection{Topology}

Figure 1 illustrates the general overview of our proposed fully optical ring $\mathrm{NoC}$ for a network of 8 nodes. It consists of a Laser source, and three waveguides that connect the nodes using a ring topology. The first waveguide is used for static communication, the second one dynamic communication, and the third as arbitration waveguide. The arbitra- 


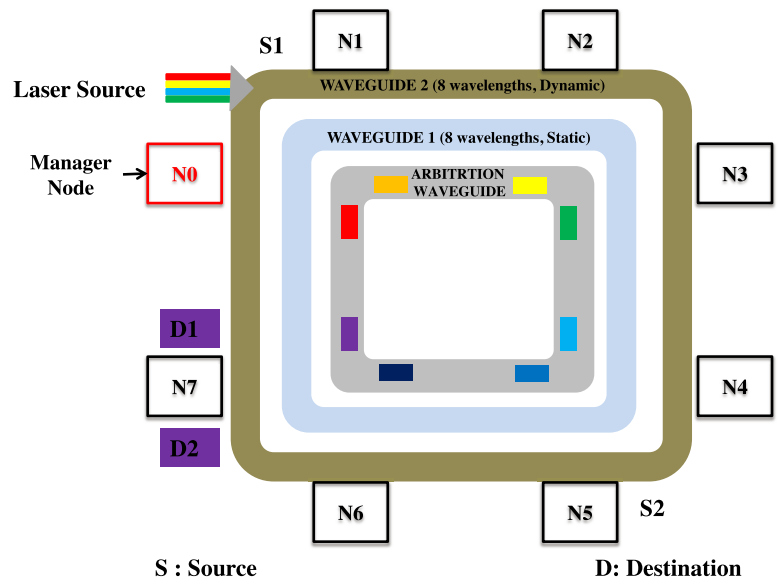

Fig. 1 Proposed architecture.

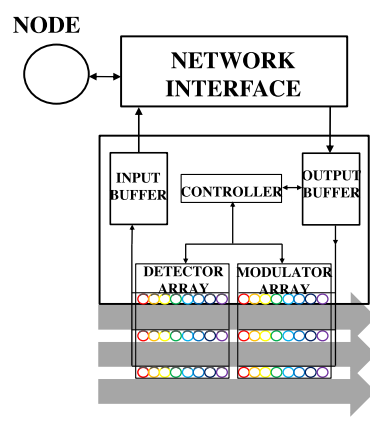

a) Normal node

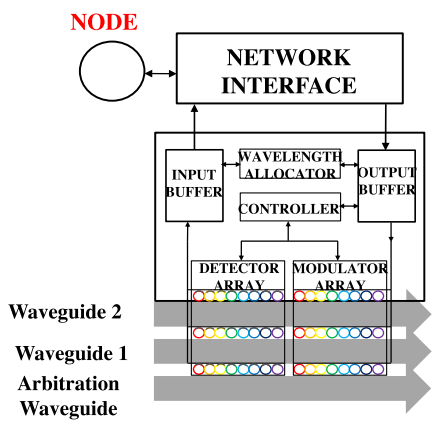

a) Manager node
Fig. 2 Nodes microarchitecture.

tion waveguide consists of the same number of wavelengthchannels with the number of nodes. A token is assigned to every wavelength-channel, each representing the right to modulate optical data intended for a particular node. In the static communication waveguide, a single wavelengthchannel is statically allocated for each destination node as receiving channel. The destination node receives optical data from a sender node by switching "ON" the detector of the wavelength-channel uniquely assigned for that particular node. The dynamic communication waveguide consists of multiple wavelengths which are shared by all nodes. Unlike the static waveguide, wavelengths are dynamically allocated by a manager node to source-destination communication pairs. The manager node is a special node, denoted NO in Fig. 1. It performs dynamic wavelength allocation based on requests in execution time.

Figures 2 (a) and (b) show microarchitecture of the normal and manager nodes, respectively. The normal node consists of electronic input and output buffers, arrays of modulators/detectors (silicon photonic devices), and a controller. The controller is used for switching state of the modulators and detectors to modulate/detect optical data stream into/from a waveguide. In addition, the manager node contains a wavelength allocator.

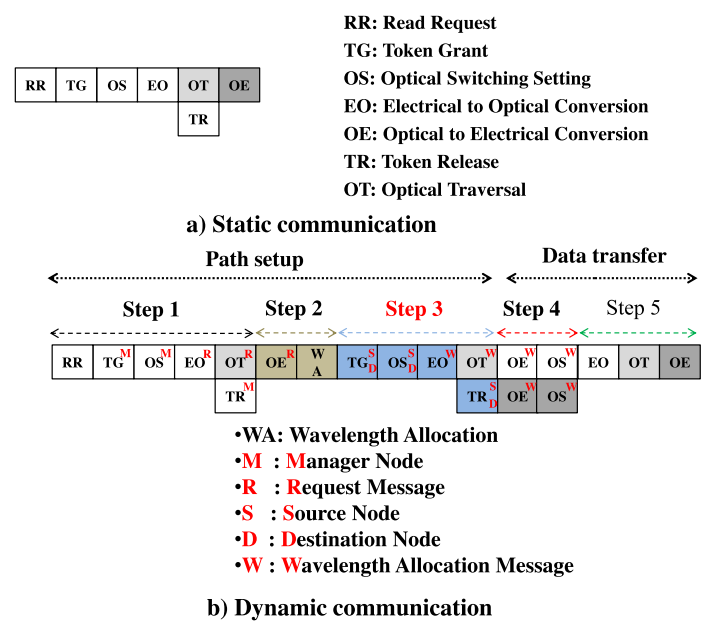

Fig. 3 Time Diagram of a) Static and b) Dynamic communications.

\subsection{Communication Mechanisms}

Our proposed architecture offers two types of communications: static and dynamic. The static communication is based on a token-based arbitration. The dynamic communication uses a manager node to allocate wavelengths to source-destination communication pairs. While static communication requires a low communication overhead, it offers only a single wavelength-channel bandwidth for data transfer. The dynamic communication on the other hand offers higher bandwidth at the cost of a higher arbitration overhead of requesting wavelength allocation to the manager node.

\subsubsection{Static Communication}

Let's consider a static communication between node $\mathrm{N} 1$ (as source S1) and node N7 (as destination D1) shown in Fig. 1. By following the communication steps of Fig. 3 (a) which shows the pipeline stages of a static communication, node N1 injects an electronic message data, to the network interface, which is saved in the node's output buffer. The controller reads its destination address (node N7) from the message header (RR). Next, a detector, associated to the wavelength for the destination node N7, is switched "ON" to grab the token for sending data on node N7's specified receiving wavelength-channel (TG). When source node N1 grabs the token, it sets up related modulator (OS) to prepare the optical data modulation. Electrical message data are modulated into optical data (EO) by node N1 and injected onto the static waveguide (node N7's receiving wavelength-channel). Then, modulated optical data are transferred on the statically assigned destination node N7's receiving wavelengthchannel (OT), and finally the grabbed token is released by the source node N1 (TR) when data modulation is completed. Destination node N7 detects the optical data transferred on the static waveguide and converts them into electronic data (OE). Note that each pipeline stage of Fig. 3 (a) 
may take multiple cycles depending on the message size and the token availability (congestion).

\subsubsection{Dynamic Communication}

Let's consider a dynamic communication between node N5 (as source S2) and node N7 (as destination D2) shown in Fig. 1. A dynamic communication is a combination of static communications (steps 1 and 3), a wavelength allocation (step 2), and data transfer (steps 4 and 5) shown in the time diagram of Fig. 3 (b). The dynamic communication can be divided into two phases as in a circuit switching communication: path setup (steps 1 to 3 ) and data transfer (step 4 and 5). First the source node N5 sends a request to the manager node N0 (Step 1, static communication in which N5 and N0 are the source and destination, respectively). When the manager node N0 receives node N5's request and there is a free path in the dynamic waveguide between source node N5 and destination node N7, the manager node allocates the path for the pair (step 2) and sends grant messages using static communication to both N5 and N7, source and destination nodes, respectively (step 3). It's important to notice that in this step, node N7 and node N5's tokens for static communication may not be available at the same time, however the grants are sent only when both tokens are grabbed by N0. After N5 and N7 nodes receive the path grant messages sent by manager node $\mathrm{N} 0$ (step 4), the source node $\mathrm{N} 5$ modulates the data to the dynamic waveguide for data transfer. Destination node N7 detects the data on the dynamic waveguide and the communication ends with a tear down message (step 5).

\subsubsection{Bended Static and Dynamic Communications}

Both static and dynamic communications may occur at the same time, in a bended way. Let's assume that, the previous communication examples of Sects.3.2.1, and 3.2.2 happen at the same time. In this case, both static and dynamic communications have the same destination node. The static communication between source node $\mathrm{N} 1$ and destination node N7 uses node N7's receiving wavelength-channel of the static communication waveguide. In the step 3 of the dynamic communication between source node N5 and destination node $\mathrm{N} 7$, the manager node $\mathrm{N} 0$ has to send the path grant message to destination node N7. Hence, it also need to use the same node N7's receiving wavelength-channel. If the static communication between N1 and N7 is still not completed when the dynamic communication between node N5 and N7 reaches the step 3; as the token for N7's receiving wavelength channel is not available, the manager node NO will delay the following step of the dynamic communication until the static communication between source $\mathrm{N} 1$ and destination $\mathrm{N} 7$ finishes. As in this example, the token based arbitration of static communication, and the manager node in dynamic communication help to solve any contention that may take place.

\section{Discussion}

A key point of our architecture is the possibility to choose between two wavelength allocation mechanisms. On one hand static allocation offers a quick establishment of communication between nodes with low data transfer bandwidth. On the other hand, dynamic allocation with high bandwidth, suffers from higher overhead of communication establishment. In this Section, we describe how we take advantage of both communication mechanisms to achieve good performance.

\subsection{Normal Selection Mechanism}

Let's assume $L a t_{\text {static }}$, and $L a t_{\text {dynamic }}$, the zero-load latencies for sending a message using the static and the dynamic allocation mechanisms, respectively.

Lat $_{\text {static }}$ can be defined by Eq. (1) as:

$$
\text { Lat }_{\text {static }}=\text { Lat }_{\text {setup static }}+\frac{\text { message }_{\text {size }}}{B W_{\text {static }}}
$$

where $L a t_{\text {setup static }}$ is the latency for path setup, and $B W_{\text {static }}$ is the bandwidth for the static allocation mechanism.

Lat dynamic can be defined by Eq. (2) as:

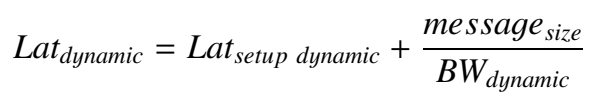

where $L a t_{\text {setup dynamic }}$, and $B W_{\text {dynamic }}$ are the path setup latency, and the bandwidth for the dynamic allocation mechanism, respectively. Although latency of static communication seems larger than the latency of dynamic communication this situation may change for certain message sizes. Because of the low bandwidth of data transfer in static communication, the data transfer time can be considerably high for large message sizes. The higher overhead of path setup in dynamic communication will no longer be a disadvantage for such cases, because of its higher data transfer bandwidth. Let's assume a communication case in which: i) both latency of static and dynamic communications are equal for a given message size; ii) a single wavelength-channel bandwidth is used for static communication while $n(n>1)$ wavelengths are used for dynamic communication, we can derive Eq. (3):

$$
\text { message }_{\text {size }}=\frac{\text { Setup }_{\text {diff }} \times n \times B W_{\text {static }}}{(n-1)}=\text { threshold }
$$

as $B W_{\text {dynamic }}=n \times B W_{\text {static }}$ and $S e t u p_{\text {diff }}$ is the setup time difference between static and dynamic communications. Equation (3) defines the threshold message size for which static communication outperforms the dynamic communication. For any value of message size higher than threshold, the dynamic communication outperforms the static communication. Using this threshold, we can classify messages as small or large. The normal selection mechanism selects between the two communication modes using the message 
size. While static allocation mechanism is selected for message sizes smaller than the threshold, the dynamic allocation mechanism is selected for higher message sizes.

\subsection{Smart Selection}

When the network is highly loaded, the latency for dynamic communication quickly increases and many dynamic communication requests have to wait for resource allocation. Under such situation, there is a trade-off between waiting for high bandwidth dynamic communication resource to be freed, and a quick establishment of low-bandwidth static communication. In order to optimize the utilization of both static and dynamic communications, we introduce a smart selection mechanism that helps to choose static or dynamic communication under the congested situations.

Manager node checks the number of waiting request messages for dynamic communication to confirm congestion. The congestion status is defined based on a threshold number of waiting request messages in the manager node. The smart selection mechanism refuses further dynamic communication requests when this threshold is reached and notifies the requester source nodes to select static communication rather than waiting a long time for the dynamic resource. We can expect that, this mechanism alleviates congestion in the dynamic communication and improves performance. Experimental results are shown in the next Section.

\section{Performance Evaluation}

We evaluate the performance of our proposed architecture using a modified version of Phoenixsim [16], a photonic NoC simulator. First the proposed architecture is compared with a hybrid photonic ring NoC we previously suggested [11], and a hybrid Mesh photonic NoC (MPNoC); in terms of arbitration energy, and performance. Then we show a performance comparison between a static wavelength allocation (static communication) and dynamic wavelength allocation (dynamic communication) techniques; for small and large size messages. A performance evaluation of our proposal under neighbor and hotspot traffic patterns is then shown for a 64-node network. For scalability issue, we show the performance of our architecture for 32 and 128 nodes under a uniform traffic pattern. Finally, we compare our architecture (referred as fully optical ring NoC (FORNoC) in the rest of the paper), to several related works in term of hardware cost.

\subsection{Simulation Setup}

Following are some simulation setups we use to evaluate the performance of our architecture:

[OREX]: Figure 4 shows an 8-node OREX NoC's topology [11]. It consists of an optical ring network and an electronic central crossbar. The optical ring is organized with two unidirectional waveguides forming

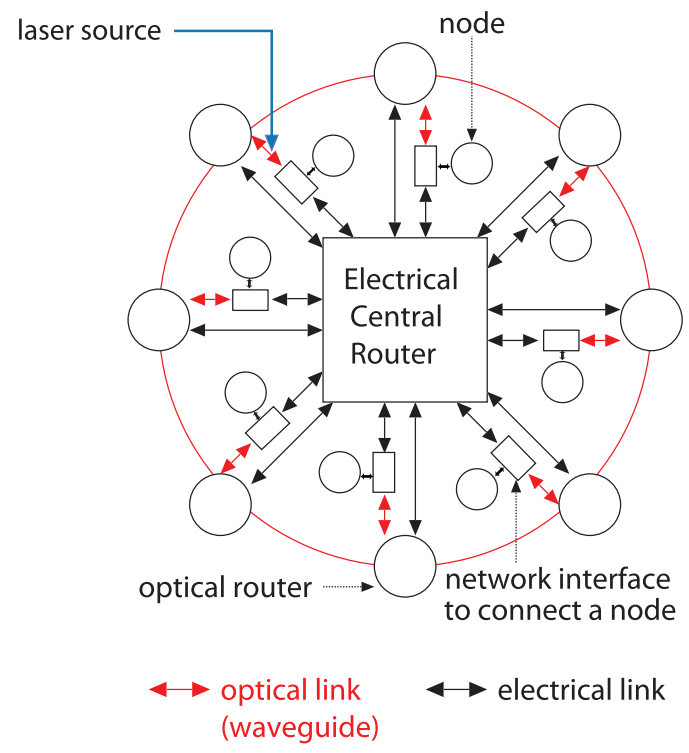

Fig. 4 OREX topology for an 8-node network.

a bidirectional link that connects the network nodes in both clockwise and counter-clockwise directions. Each waveguide consists of multiple wavelengths. To communicate, a source node sends a path setup request to the central crossbar. The central crossbar allocates a path (preferentially the shortest) when available and sends back path allocation notification to the source and destination nodes. Then data transfer takes place. After data transfer finishes, the source node sends a request to the central crossbar for the optical path teardown. OREX has the advantage of shorten path setup, and teardown latencies because all network nodes are directly connected to the central crossbar which performs the optical path allocation. For fair comparison with FORNoC, we use the same number of communication waveguides for both architectures. While FORNoC uses static and dynamic communication waveguides, OREX uses both waveguides (clockwise and counter-clockwise) for dynamic communication.

[MPNoC]: The MPNoC is a hybrid NoC proposed in [7] with mesh topology. The network is formed by a mesh optical NoC overlaid by a similar mesh electronic path setup network.

[Message Size]: We use two different message sizes: 20 for small size message, and 400 Bytes for large size message. A node randomly generates either 20 or 400 Bytes size messages. These two sizes are selected based on consideration in Sect. 4.

[Network size and wavelengths]: As our architecture uses a single wavelength-channel per node for the static communication, the number of required wavelengths for static communication is proportional to the number of nodes in the network. The same number of wavelength is also required for the arbitration. Thus to implement 32-node network, we use two waveguides with 
Table 1 Simulation parameters.

\begin{tabular}{l|l}
\hline Parameter & Setting \\
\hline NoC Architecture & FORNoC, OREX, MPNoC \\
\hline Number of Nodes & $32,64,128$ \\
\hline Traffic Patterns & Uniform, neighbor, hotspot \\
\hline Message Sizes & 20 Bytes, 400 Bytes \\
\hline $\begin{array}{l}\text { Communication Channel \# wavelength } \\
\times \# \text { waveguide }\end{array}$ & $32 \times 2,64 \times 2,64 \times 3$ \\
\hline Clock Frequency & $5 \mathrm{GHz}[7]$ \\
\hline Speed of Modulation & $10 \mathrm{Gbps}[9],[15]$ \\
\hline Communication Types for FORNoC & $\begin{array}{l}\text { Static only, dynamic only, } \\
\text { and combination }\end{array}$ \\
\hline
\end{tabular}

each of them using only 32 wavelengths per waveguide for static, and arbitration waveguides, respectively and a third waveguide with 64 wavelengths for dynamic communication. As most of previous works suggest using a maximum of 64 wavelengths per waveguide, for the case of 128 nodes, we used two waveguides of 64 wavelengths each to connect the node statically as well as to perform arbitration; meanwhile a single waveguide of 64 wavelengths is used for the dynamic communication. Hence our architecture requires a total of 5 waveguides to implement 128 nodes.

[Smart selection threshold]: The smart selection threshold depends on several experimental parameters. In the experimental conditions of the paper, based on simulations, we use 35 waiting dynamic communication requests for the smart selection threshold. Further requests for dynamic communication are directed to use the static communication.

[Clock Frequency and Speed of Modulation]: For the clock frequency, we use $5 \mathrm{GHz}$ as used in [7], [10], [13]. Although 12.5Gbps [10], 40Gbps [7] modulation speeds have been suggested, we use 10Gbps as in [9], [15] for our simulations.

[Measurement]: The communication latency is measured as the time to transfer the whole message, from when it is created to when the message reaches it destination. We evaluate the average latency and average bandwidth of the networks as a function of the message injection rate during a simulation time. The average network latency/bandwidth, for an injection rate is depicted as the average latency/bandwidth of all messages that reach their destinations during the simulation time.

Table 1 summarizes our simulation parameters.

\subsection{Simulation Results and Analysis}

\subsubsection{Performance Comparison with Hybrid NoCs}

(1) Comparison of Energy Consumption for Arbitration

In our simulations, design parameters such as static and dynamic energy of every component are integrated. Energy consumed for injection, ejection arbitration, buffering opto-electrical conversions, data transfer are calculated

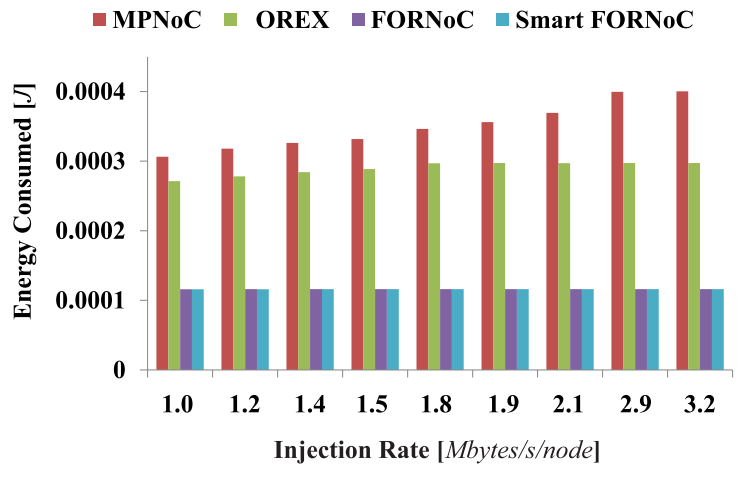

Fig. 5 Comparison of the arbitration energy consumption.

during simulation execution time. The main energy consumption difference between the hybrid NoCs (MPNoC, OREX) and FORNoC is the arbitration energy as the hybrid NoCs perform the arbitration electronically. For a given injection rate we depicted the average portions of energy consumed in the network for path arbitration for MPNoC, OREX and FORNoC. Figure 5 shows the average energy consumed for the networks versus the injection rate under a uniform random traffic pattern. The results show that MPNoC and OREX consume high arbitration energy as compared to the proposed FORNoC networks. Both MPNoC and OREX consume higher energy by exchanging control messages between source and destination via electronic path setup networks whereas FORNoC performs those tasks optically. FORNoC with smart selection consumes nearly similar amount of energy as the FORNoC with normal selection because only fewer overheads are added to the arbitration which in turn reduces the energy consumed with an improvement in path allocation. Because power constraint is so severe in future NoCs, FORNoC can be an alternative low power solution.

\section{(2) Comparison of Latency and Bandwidth}

Figure 6 shows the performance of MPNoC, OREX and FORNoC in terms of latency (a), and bandwidth (b) under uniform random traffic. OREX outperforms MPNoC and FORNoC with both selection mechanisms in average latency and bandwidth. The OREX has a low latency path setup network (electronic crossbar) which explains its latency and bandwidth performances. MPNoC with a larger electronic path setup network performs almost similarly to the FORNoC in terms of latency and bandwidth. The FORNoC with smart selection slightly outperforms MPNoC and normal FORNoC by relaxing the network congestion.

\subsection{Comparison between Static and Dynamic Communi- cations}

Figure 7 shows the simulation results for static and dynamic communications standing alone under uniform traffic pattern for 20, and 400 Bytes message sizes. These results confirm our assumption in Sect. 4 that, for small message size 


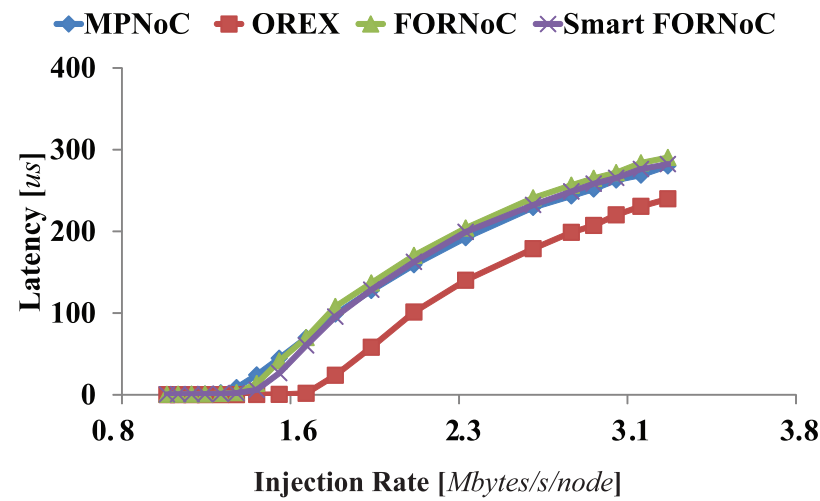

a) Latency

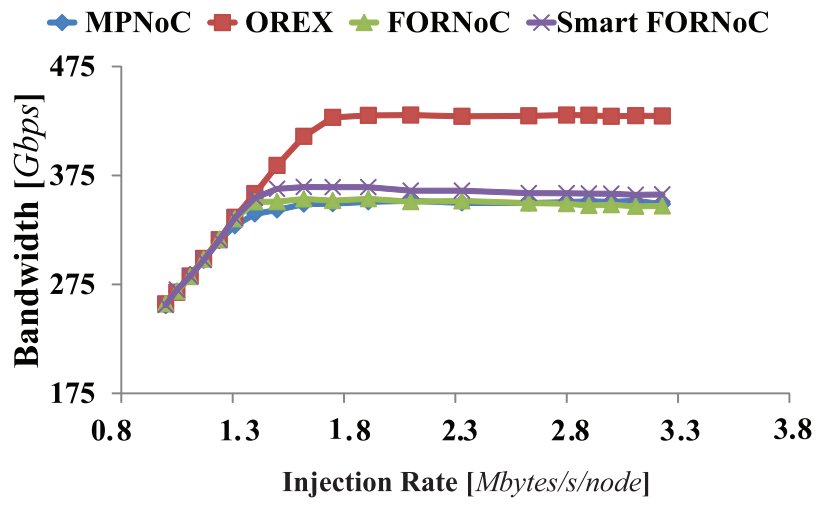

b) Bandwidth

Fig. 6 Performance comparison under uniform traffic.

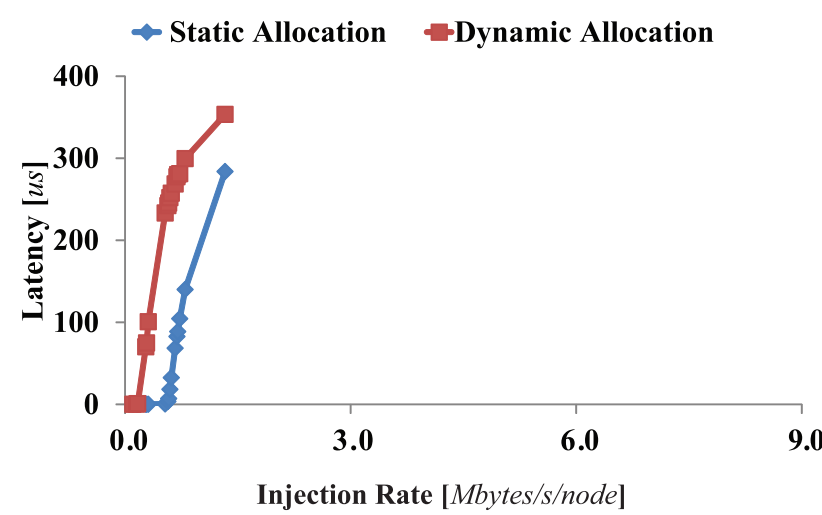

a) 20 Bytes message size.

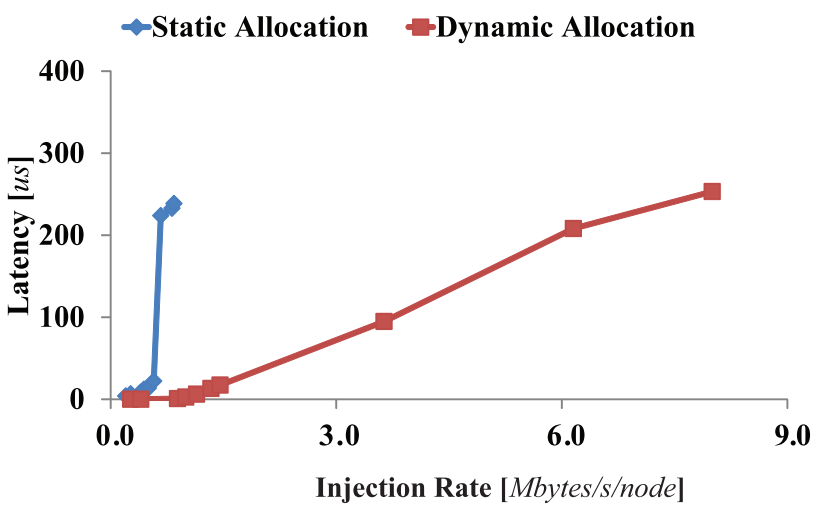

b) 400 Bytes message size.

Fig. 7 Average latencies for static and dynamic communications under uniform random traffic.

(20 Bytes), the static communication outperforms the dynamic one. Because of the small message sizes, the fast path latency of the static communication is preferable (Fig. 7 (a)). In the other hand, for larger message sizes, higher bandwidth is more efficient than slower path setup. As shown in Fig. 7 (b), dynamic communication performs better in such case.

\subsubsection{Performance under Partially Localized and Local- ized Probabilistic Traffic Patterns}

In opposition to the uniform random traffic pattern in which communication is uniformly distributed throughout the network, we also evaluate performance of our architecture for partially and localized traffic patterns.

\section{(1) Partially Localized Traffic Pattern}

We implement neighbor communication pattern in which nodes communicate with their neighboring left and right node in a random manner. Figure 8 shows the performance in terms of latency (a), and bandwidth (b) for the normal and smart allocation mechanisms. As stated in Sect. 4, the smart selection further improves the performance of the selection based only on message size by using the network congestion information, to reduce contention for network resources.

\section{(2) Localized Traffic Pattern}

For localized traffic, we use a hotspot traffic pattern. A node is randomly chosen as hotspot node, and all other nodes communicate with that node. Figure 9 shows the performance in terms of latency (a) and bandwidth (b), for the normal and smart allocation mechanisms. Because network traffic is not distributed in the network, smart and normal selection mechanisms perform almost similarly. For higher injection rate, either static and dynamic paths to the hotspot node are constantly busy, hence the smart selection degrades the performance by inquiring extra arbitration at the hotspot to allocate communication paths.

\subsubsection{Scalability}

Figure 10 shows the latency versus the injection rate for 32,64 , and 128-node networks under uniform traffic pattern for normal, and smart selection mechanisms. Although for larger networks: i) average distance is longer (more network nodes); ii) network saturates with smaller load because disjoint paths on the ring are reduced, the results show that 


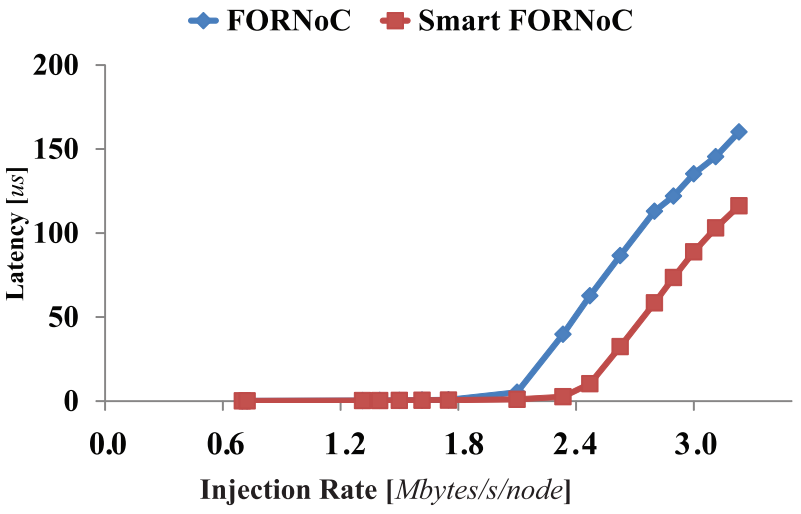

a) Latency

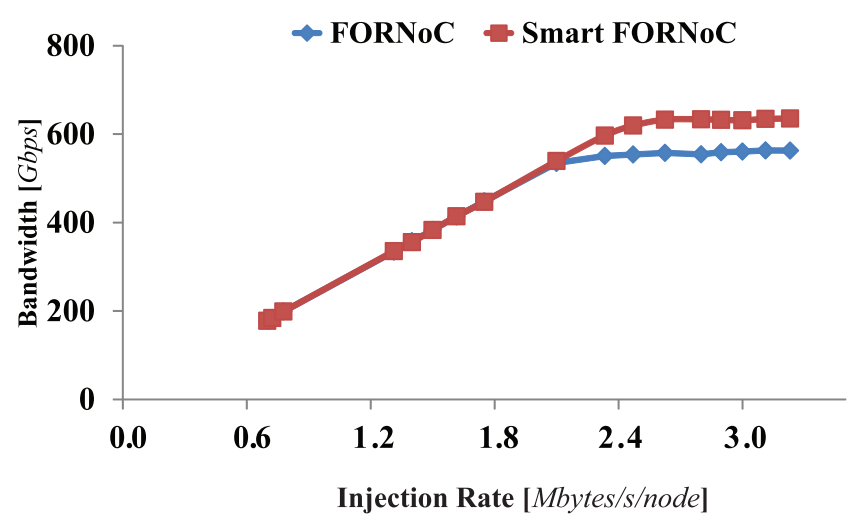

b) Bandwidth

Fig. 8 FORNoC vs. smart FORNoC performance for 64-node under neighbor traffic.

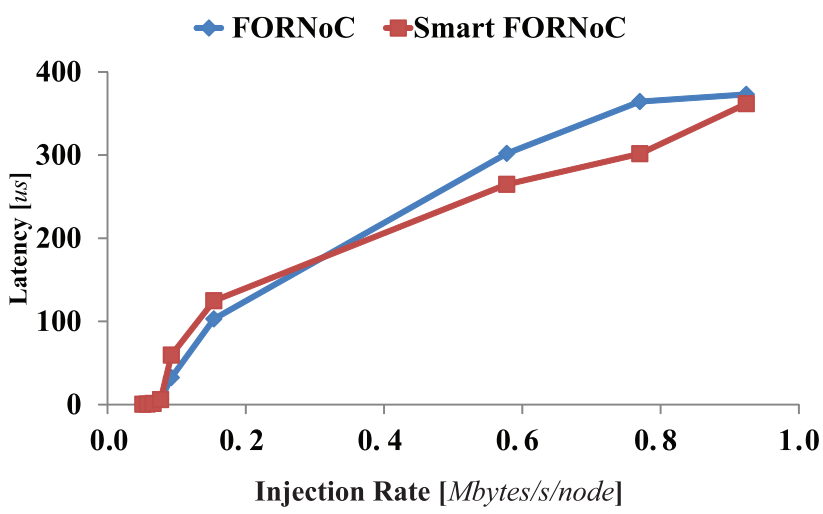

a) Latency

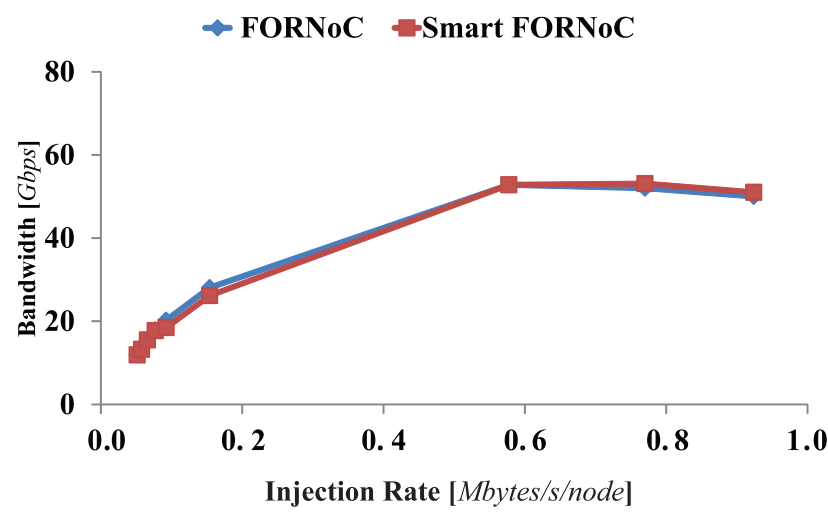

b) Bandwidth

Fig. 9 FORNoC vs. smart FORNoC performance for 64-node under hotspot traffic.

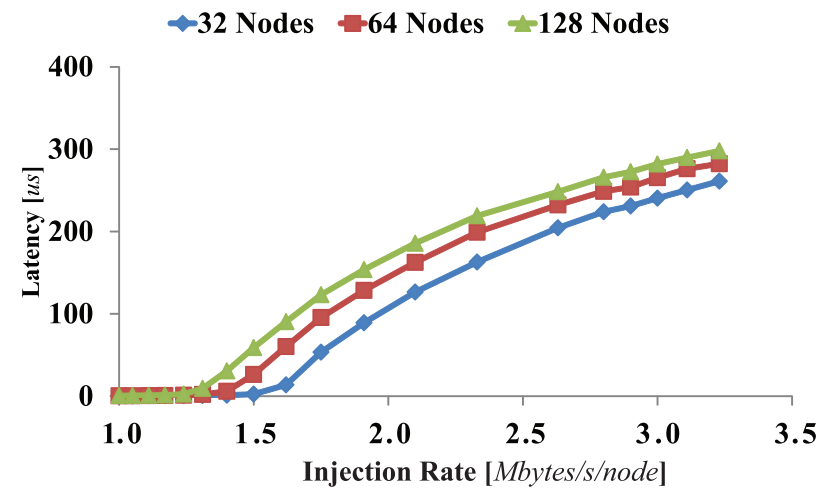

a) Normal Selection

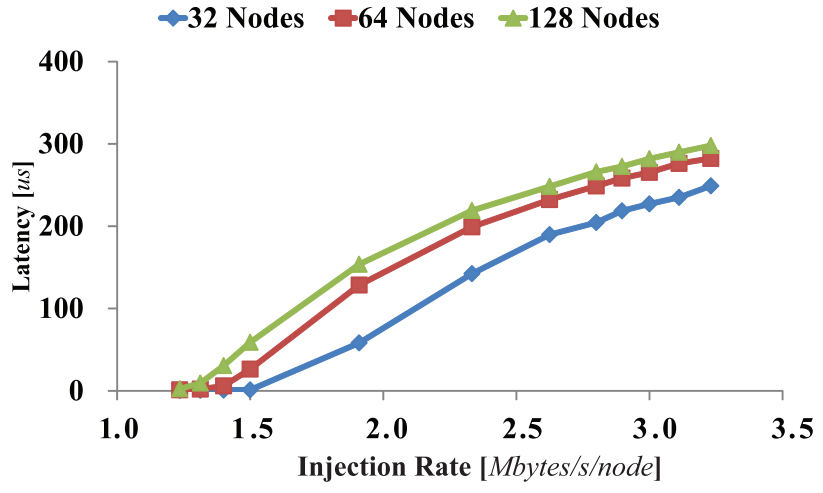

b) Smart Selection

Fig. 10 Average latencies for 32, 64, and 128-node under uniform random traffic.

the performance of FORNoC is scalable for both selection mechanisms.

\subsection{Architecture Hardware Cost Comparison}

To build a 64-node network, our architecture uses a total of 16256 ring resonators (254 per node, 126 for static waveguide, 126 for arbitration waveguide, and 2 for the dynamic waveguide), 3 waveguides (static, dynamic, and arbitration), 8256 photodetectors (127 per node, 63 for static waveguide, 63 for arbitration waveguide, and 1 for dynamic waveguide). Table 2 summarizes the hardware cost requirements of a 64-node for a hybrid 2- $D$ planar (mesh,torus) networks [7], PROPEL [9], OREX [11], CORONA [13], and 
Table 2 Architecture hardware cost comparison for 64-node networks.

\begin{tabular}{l|c|c|c|c|c}
\hline & 2-D Hybrid (Mesh, Torus) [7] & PROPEL [9] & OREX [11] & CORONA [13] & FORNoC \\
\hline Wavelengths & 64 & 64 & 64 & 64 & 64 \\
\hline Waveguides & 64 & 64 & 2 & 64 & 3 \\
\hline Ring Resonators & 1024 & 3072 & 256 & 72192 & 16256 \\
\hline Photodetectors & 4096 & 1536 & 8192 & 7424 & 8128 \\
\hline Electrical Switches & $5 \times 5(64)$ & $5 \times 5(16)$ & $64 \times 64(1)$ & - & - \\
\hline
\end{tabular}

FORNoC. Compared to hybrid architectures, fully optical FORNoC and CORONA networks do not require electronic switches. The fully optical networks however use more optical components necessary for arbitration. FORNoC uses fewer optical waveguides and ring resonators compared to CORONA by providing both static and dynamic wavelength allocation techniques. CORONA however uses fewer photo detectors (less dedicated paths).

\section{Conclusion and Future Works}

In this paper, we have proposed a fully scalable photonic ring NoC, which combines static and dynamic wavelength allocation communication mechanisms. The architecture takes advantage of both low-overhead/low-bandwidth of static, and high-overhead/high-bandwidth dynamic communications using wavelength allocation selection techniques, based on message size (normal selection), and congestion information (smart selection).

Performance evaluation results under various probabilistic traffic patterns show that our proposed fully optical ring network FORNoC presents a good performance for both wavelength allocation selection techniques. We also showed that our architecture reduces by more than half, the energy consumption necessary for arbitration compared to hybrid ring and mesh NoCs. A comparison with other previous work in term of architecture hardware cost shows that our architecture can be an attractive cost-performance efficient interconnection infrastructure for future SoCs and CMPs.

In the future we would like to investigate the performance of the architecture with multiple data-stream dynamic communication. Allowing the division of the wavelength of the dynamic wavelength allocation waveguide into two, three or four groups of wavelengths may reduce the dynamic bandwidth, however an improvement in performance can be expected due to congestion reduction. Another improvement of this work is to investigate fault tolerance technique for FORNoC and evaluate its performance using real applications.

\section{Acknowledgment}

This research is supported in part by NII Joint research and Grants-in-Aid for Scientific Research of Japan Society for Promotion of Science (JSPS), No.22500042.

\section{References}

[1] Q. Xu, S. Manipatruni, B. Schmidt, J. Shakya, and M. Lipson,
" $12.5 \mathrm{Gbit} / \mathrm{s}$ carrier-injection-based silicon micro-ring silicon modulators," Opt. Express, vol.15, no.2, pp.430-436, Jan. 2007.

[2] I. Young, E. Mohammed, J. Liao, A. Kern, S. Palermo, B. Block, M. Reshotko, and P. Chang, "Optical I/O technology for tera-scale computing," IEEE Int. Solid-State Circuits Conf. Digest of Technical Papers, 2009. ISSCC 2009. pp.468-469, 469a, Feb. 2009.

[3] M. Reshotko, B. Block, B. Jin, and P. Chang, "Waveguide coupled Ge-on-oxide photodetectors for integrated optical links," 2008 5th IEEE Int. Conf. Group IV Photonics, pp.182-184, Sept. 2008.

[4] C. Holzwarth, J. Orcutt, H. Li, M. Popovic, V. Stojanovic, J. Hoyt, R. Ram, and H. Smith, "Localized substrate removal technique enabling strong-confinement microphotonics in bulk Si CMOS processes," Lasers and Electro-Optics, 2008 and 2008 Conf. Quantum Electronics and Laser Science, CLEO/QELS 2008, pp.1-2, May 2008.

[5] M. Dahlem, M. Popovic, C. Holzwarth, A. Khilo, T. Barwicz, H. Smith, F. Kartner, and E. Ippen, "Electronic-photonic integrated circuits in silicon-on-insulator platforms," General Assembly and Scientific Symposium, 2011 XXXth URSI, p.1, Aug. 2011.

[6] N. Kirman, M. Kirman, R.K. Dokania, J.F. Martinez, A.B. Apsel, M.A. Watkins, and D.H. Albonesi, "Leveraging optical technology in future bus-based chip multiprocessors," Proc. 39th Annual IEEE/ACM International Symposium on Microarchitecture, MICRO 39, Washington, DC, USA, pp.492-503, 2006.

[7] A. Shacham, K. Bergman, and L. Carloni, "Photonic networks-onchip for future generations of chip multiprocessors," IEEE Trans. Comput., vol.57, no.9, pp.1246-1260, Sept. 2008.

[8] S. Koohi and S. Hessabi, "Contention-free on-chip routing of optical packets," 3rd ACM/IEEE International Symposium on Networkson-Chip, NoCS 2009, pp.134-143, May 2009.

[9] R. Morris and A. Kodi, "Exploring the design of 64- and 256-core power efficient nanophotonic interconnect," IEEE J. Sel. Top. Quantum Electron., vol.16, no.5, pp.1386-1393, Sept.-Oct. 2010.

[10] Y. Pan, P. Kumar, J. Kim, G. Memik, Y. Zhang, and A. Choudhary, "Firefly: Illuminating future network-on-chip with nanophotonics," Proc. 36th Annual International Symposium on Computer Architecture, ISCA ’09, New York, NY, USA, pp.429-440, ACM, 2009.

[11] A.D.A. Cisse, P. Qiu, H. Irie, T. Miyoshi, and T. Yoshinaga, "Orex: A hybrid photonic network-on-chip of optical ring and electrical crossbar," Proc. International Workshop on Innovative Architecture for Future Generation High-Performance Processors and Systems (IWIA), 2010.

[12] M. Briere, B. Girodias, Y. Bouchebaba, G. Nicolescu, F. Mieyeville, F. Gaffiot, and I. O'Connor, "System level assessment of an optical $\mathrm{NoC}$ in an mpsoc platform," Design, Automation Test in Europe Conference Exhibition, DATE '07, pp.1-6, April 2007.

[13] D. Vantrease, R. Schreiber, M. Monchiero, M. McLaren, N. Jouppi, M. Fiorentino, A. Davis, N. Binkert, R. Beausoleil, and J. Ahn, "Corona: System implications of emerging nanophotonic technology,' ISCA '08, 35th International Symposium on Computer Architecture, pp.153-164, June 2008.

[14] C. Li, M. Browning, P.V. Gratz, and S. Palermo, "Luminoc: A power-efficient, high-performance, photonic network-on-chip for future parallel architectures," Proc. 21st International Conference on Parallel Architectures and Compilation Techniques, PACT '12, New York, NY, USA, pp.421-422, ACM, 2012.

[15] S. Koohi and S. Hessabi, "All-optical wavelength-routed architec- 
ture for a power-efficient network on chip," IEEE Trans. Comput., vol.PP, no.99, pp.1-14, 2012.

[16] J. Chan, G. Hendry, A. Biberman, K. Bergman, and L. Carloni, "Phoenixsim: A simulator for physical-layer analysis of chip-scale photonic interconnection networks," Design, Automation Test in Europe Conference Exhibition (DATE), 2010, pp.691-696, March 2010.

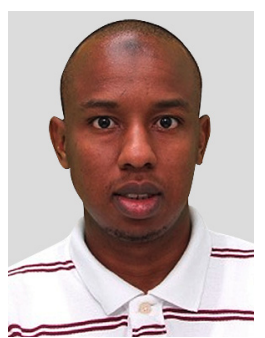

Ahmadou Dit Adi Cisse received his B.E. degree from the Faculty of Science of Sfax, Tunisia in 2005. Since Oct. 2007 he has been with the Graduate School of Information Systems, The University of ElectroCommunications where he received his M.E. degree in Sept. 2009. From Oct. 2009 he enrolled as Ph.D. candidate. His research interests include Interconnection network for Network-onChip in general and Optical Network-on-Chip in particular. He is an IEEE student member.

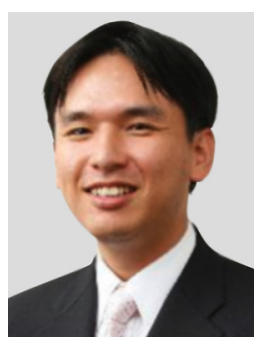

Michihiro Koibuchi received the B.E., M.E., and Ph.D. degrees from Keio University, Yokohama, Japan, in 2000, 2002, and 2003, respectively. He was a visiting researcher at the Technical University of Valencia, Spain, in 2004 and a visiting scholar at the University of Southern California, in 2006. He is currently an associate professor in the Information Systems Architecture Research Division, National Institute of Informatics, Tokyo, and the Graduate University for Advanced Studies, Japan. His research interests include the areas of high-performance computing and interconnection networks. He is a member of the IEICE, IPSJ, and IEEE.

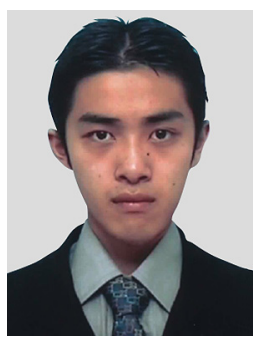

Masato Yoshimi recieved the B.E., M.E., and Ph.D. degrees from Keio University, Japan, in 2004, 2006, and 2009, respectively. From 2009 to September 2012, he was an assistant professor of Doshisha University. Since October 2012, he has been an assistant professor in Graduate School of Information Systems, University of Electro-Communications. His research interests include the areas of intelligent systems, reconfigurable computing and parallel processing.

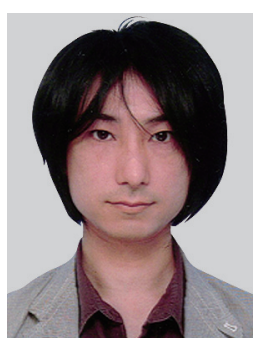

Hidetsugu Irie is an associate professor of network computing at Graduate School of Information Systems, the University of ElectroCommunications. His research interests include microarchitectures, and network systems. He has a Ph.D. in Information Science and Technology from The University of Tokyo. He was a researcher of Japan Science and Technology Agency from 2004 to 2008, and was an assistant professor of The University of Tokyo from 2008 to 2010. He is a member of ACM, IEEE, IEICE,

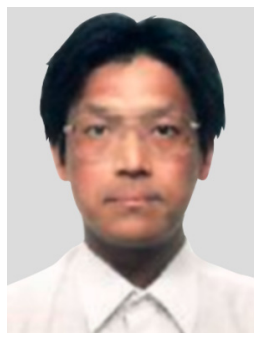

Tsutomu Yoshinaga received his B.E., M.E., and D.E. degrees from Utsunomiya University in 1986, 1988, and 1997, respectively. From 1988 to July 2000, he was a research associate of Faculty of Engineering, Utsunomiya University. He was also a visiting researcher at Electro-Technical Laboratory from 1997 to 1998. Since August 2000, he has been with the Graduate School of Information Systems, UEC, where he is now a professor. His research interests include computer architecture, interconnection networks, and network computing. He is a member of ACM, IEEE, and IPSJ.

and IPSJ. 